\title{
Designing screening protocols for amphibian disease that account for imperfect and variable capture rates of individuals
}

\author{
Stefano Canessa, ${ }^{1,3}$ An Martel, ${ }^{2}$ and Frank Pasmans ${ }^{2}$ \\ ${ }^{1}$ School of Botany, University of Melbourne, Victoria 3010 Australia \\ ${ }^{2}$ Faculty of Veterinary Medicine, Ghent University, Salisburylaan 133, Merelbeke 9820 Belgium
}

\begin{abstract}
The amphibian chytrid fungus, Batrachochytrium dendrobatidis, is one of the main factors in global amphibian decline. Accurate knowledge of its presence and prevalence in an area is needed to trigger conservation actions. However, imperfect capture rates determine the number of individuals caught and tested during field surveys, and contribute to the uncertainty surrounding estimates of prevalence. Screening programs should be planned with the objective of minimizing such uncertainty. We show how this can be achieved by using predictive models that incorporate information about population size and capture rates. Using as a case study an existing screening program for three populations of the yellow-bellied toad (Bombina variegata pachypus) in northern Italy, we sought to quantify the effect of seasonal variation in individual capture rates on the uncertainty surrounding estimates of chytrid prevalence. We obtained estimates of population size and capture rates from mark-recapture data, and found wide seasonal variation in the individual recapture rates. We then incorporated this information in a binomial model to predict the estimates of prevalence that would be obtained by sampling at different times in the season, assuming no infected individuals were found. Sampling during the period of maximum capture probability was predicted to decrease upper $95 \%$ credible intervals by a maximum of $36 \%$, compared with least suitable periods, with greater gains when using uninformative priors. We evaluated model predictions by comparing them with the results of screening surveys in 2012. The observed results closely matched the predicted figures for all populations, suggesting that this method can be reliably used to maximize the sampling size of surveillance programs, thus improving their efficiency.
\end{abstract}

Key words: Batrachochytrium dendrobatidis; Bayesian statistics; binomial model; Bombina variegata pachypus; chytridiomycosis; detectability; Jolly-Seber model; Liguria; mark-recapture; monitoring; predictive models; surveillance.

\section{INTRODUCTION}

Chytridiomycosis, a disease caused by the amphibian fungus Batrachochytrium dendrobatidis, is among the main drivers of global amphibian decline (Berger et al. 1998, Fisher et al. 2009). Clarifying the role of $B$. dendrobatidis in the decline of species can help in adjusting conservation strategies; in fact, optimal responses may differ, depending on the relative importance of $B$. dendrobatidis and other threats (such as habitat loss). Chytridiomycosis might not be effectively mitigated by programs aimed at, for example, habitat restoration, and specific actions may be required, such as establishing captive rescue populations (Gagliardo et al. 2008).

Accurate knowledge about the presence of the pathogen is needed to inform such conservation protocols. It is therefore necessary to design and implement surveillance strategies to estimate and update the local prevalence of the pathogen (the proportion of

Manuscript received 18 January 2013; revised 10 June 2013; accepted 16 August 2013; final version received 20 October 2013. Corresponding Editor: T. W. J. Garner.

33E-mail: canessas@unimelb.edu.au individuals that are infected). Surveillance is particularly important, since outbreaks and infections can occur at sites where the habitat is not visibly degraded (Berger et al. 1998). Populations of species believed to be at risk from chytridiomycosis should therefore be screened for B. dendrobatidis to ascertain the occurrence and prevalence of the pathogen, and ultimately to determine its effect on populations.

Therefore, screening programs need to provide the best possible information in order to inform management decisions. In the case of $B$. dendrobatidis, surveillance can be problematic, since its prevalence in infected populations has been shown to vary significantly in time, possibly reflecting seasonality in environmental conditions, such as temperature (Kriger and Hero 2007). In addition, for populations of wild animals, the probability of detecting and capturing individuals is generally less than one (MacKenzie and Kendall 2002, Schmidt 2004). Only a limited proportion of any given population may actually be tested, and infected individuals might be missed, reducing the sample size, leading to uncertain estimates of prevalence, and complicating decision making. Any information that can improve the accuracy of prevalence estimates should be incorporated 
both into the planning phase of surveillance programs, and into the interpretation of the results.

As testing techniques become cheaper and more widely available, targeted screening programs could be carried out at the local scale, for example, by managers of individual protected areas. For endangered species with known small local populations, significant information can be collected with limited effort, and surveillance programs for early detection of infection can be implemented. Budget limitations often impede this type of planning; however, statistical techniques can be used to optimize surveillance programs, and to maximize the accuracy of prevalence estimates for a given budget and constraints. The usefulness of accounting for imperfect detection in surveillance programs has been demonstrated for quarantine (Burgman et al. 2009) and detection of invasive species (Garrard et al. 2008), and recent studies have highlighted the need for its consideration in wildlife disease studies (McClintock et al. 2010, Lachish et al. 2012).

In this study, we investigated how explicitly incorporating estimates of the probability of capturing individuals for testing can be used to maximize sample sizes and reduce sampling uncertainty when designing a surveillance program for chytrid. We used as a case study an ongoing screening program for the endangered Apennine yellow-bellied toad, Bombina variegata pachypus, in northern Italy. We used mark-recapture modeling to estimate population sizes and individual probabilities of capture, and a binomial model to estimate chytrid prevalence from survey results for 2011. Working in a Bayesian framework, we then combined estimates of pathogen prevalence and of seasonal variation in the probability of detection in order to develop a plan for future surveillance of the species, using different prior beliefs and single or repeated surveys. We predicted the estimates of prevalence for the 2012 season and compared them retrospectively with the actual estimates from those surveys, to assess the validity of the model in guiding survey planning.

\section{Methods}

\section{Statistical framework}

The accuracy of a screening protocol depends on the prevalence of the pathogen in the target population (the probability that any given individual is infected), and on the proportion of the total population that is sampled. When $n$ individuals are captured and tested, the number $y$ of positive results can be expressed as a binomially distributed random variable

$$
y \sim \operatorname{bin}(p, n)
$$

where the probability of success $p$ corresponds to the prevalence of the pathogen. If $p=1$, then sampling one individual is sufficient to detect the pathogen; conversely, if $p=1 / n$, the estimate of prevalence will be uncertain until all $n$ individuals are sampled. Working in a
Bayesian framework, prevalence can be described by a beta-distributed prior

$$
p \sim \operatorname{beta}(\alpha, \beta)
$$

and this formulation can be intuitively modelled in Bayesian statistical software such as WinBUGS (Lunn et al. 2000) or JAGS (Plummer 2003).

For post hoc analyses of screening results, both $y$ and $n$ (the sample size) are data points. However, when planning surveillance programs, it is necessary to consider the value that the sample size $n$ might take, as a larger sample will reduce uncertainty when estimating $p$. The value of $n$ will be determined by another binomial process

$$
n \sim \operatorname{bin}(N, c)
$$

where $N$ is the size of the screened population and $c$ the individual probability of capture. If both parameters are known or can be estimated, they can then be used to predict the sample size $n$ that can be obtained by using a given surveillance strategy (for a range of possible values of $y$ ), and to evaluate the expected accuracy of the resulting estimate of $p$.

Assuming a binomially distributed number of positive samples, increasing the probability of capture will increase the chance of detecting at least one infected individual, and increase confidence in the resulting estimate of infection prevalence. While the probability of infection is usually beyond human control, it is possible to increase the probability of detection by selecting the best survey timing and methods, and by further repeating screening sessions to increase the cumulative proportion of the population sampled. This can be calculated as

$$
\hat{C}_{\text {tot }}=1-\prod_{\mathrm{i}=1}^{\mathrm{s}}\left(1-\hat{c}_{i}\right)
$$

where $\hat{C}_{\text {tot }}$ is the total probability of capture, $\hat{c}_{i}$ is the estimated probability of capture for screening session $i$, and $s$ is the number of repeated surveys.

\section{Study species}

Chytridiomycosis is among the hypothesized causes of the decline of the Apennine yellow-bellied toad Bombina variegata pachypus, a small amphibian endemic to peninsular Italy (Guarino et al. 2007). This species is listed as endangered in the IUCN Red List (as Bombina pachypus; Andreone 2009), and is protected by the Habitats Directive of the European Union (92/43/EEC). Populations in the north and center of the species' range have declined in both number and abundance since the mid-1990s (Barbieri et al. 2004). A recent study has found evidence of infection by $B$. dendrobatidis on specimens across the Italian peninsula (Canestrelli et al. 2013). Other subspecies of Bombina variegata are prone to infection by $B$. dendrobatidis (Spitzen-van der Sluijs et al. 2011, Sztatecsny and Glaser 2011), and mortality 
resulting from chytridiomycosis has been recorded for B. v. pachypus originating from two populations in the northeastern Apennines (Stagni et al. 2004). In the region of Liguria, at the northwestern limit of the species' range, $50 \%$ of the known populations of $B$. $v$. pachypus have disappeared between 2005 and 2010; however, the role of chytrid in regional declines has never been investigated.

A screening program for known populations in the region was carried out in 2011 and 2012 to clarify the extent and magnitude of possible infections: during this program, a total of 86 and 143 individuals were caught and swabbed at eight populations in the region in 2011 and 2012, respectively (full description of sampling and diagnostic methods in Canessa et al. 2013a). In 2011, swabs were collected during a single visit to each site in late July. In 2012, each site was revisited once in June (with the exception of population 3) and three times in late July within a two-week period to minimize fluctuations in population size. All individual swabs tested negative for chytrid in both seasons.

Continuous surveillance is needed to ensure that estimates of prevalence can be reliably used to infer pathogen outbreaks. However, the activity patterns for this species are likely to influence the probability of recapture across the seasonal window for sampling. This in turn will determine the number of individuals sampled, and influence the quality of prevalence estimates. Consequently, detailed planning is needed to maximize the quality of the inference that can be made, given the limited resources available, in particular regarding the timing of surveys. For this study, we focused on the three largest known populations of $B . v$. pachypus in the Liguria region, all located in the Lavagna river catchment, over an area of approximately $24 \mathrm{~km}^{2}$.

\section{Initial estimates of pathogen prevalence}

As the first step of our analysis, we sought to obtain estimates of prevalence from the 2011 data described in Canessa et al. $(2013 a)$. We implemented the post hoc binomial model (Eq. 1-2) in JAGS (Plummer 2003), using an uninformative beta-distributed prior for prevalence (see Supplement). We ran the model to obtain 100000 samples on three separate Markov chains, after discarding the first 30000 as a burn-in and applying a thinning rate of 10 . We checked convergence by visual inspection of individual chains, and by using the Gelman-Brooks-Rubin convergence statistic (Brooks and Gelman 1998).

\section{Recapture rates: data collection and analysis}

To inform the planning of future surveys, we then sought to estimate the parameters of interest for the binomial capture process (Eq. 3); the individual probability of capture and the size of the screened populations of our study species. To do so, we collected mark-recapture data from the three target populations.
During the breeding season (April-September) in 2010 and 2011, the senior author and one of a number of field assistants visited each site between six and 14 times, with a minimum interval of one week between visits. During each survey, individuals were captured by hand, photographed (to record their unique ventral pattern), sexed by visual inspection of male nuptial pads, and then released at the point of capture. To minimize the chance of spreading pathogens, latex disposable gloves were worn during capture and handling, and replaced for each individual. Equipment and boots were also disinfected between sites (R. Speare et al. unpublished manuscript), and sites within different catchments were never sampled during the same day.

We fitted a Jolly-Seber model to the recapture histories, working in a Bayesian framework (Kéry and Schaub 2011; see Supplement). Although the species in the Liguria region is following a marked declining trend, no clear sign of decline in numbers has been observed in the three target populations since 2001, with only normal variability being observed (Canessa et al. $2013 b$ ). We therefore assumed a constant survival rate for our study period, with a semi-informative prior (uniformly distributed between 0.6 and 1) on the basis of expert knowledge and published information for this relatively long-lived species (Barandun et al. 1997, Hartel et al. 2007). We assumed a constant probability of entry, reflecting the limited dispersal observed in $B$. variegata (Barandun and Reyer 1998, Hartel 2008), using an informative prior (a uniform distribution between 0 and 0.05 ). We excluded juveniles of the year from the analysis, since individuals of this age class have not developed a full belly pattern and thus cannot be reliably marked. Finally, we estimated the daily individual probability of recapture as date-specific across the sampling season. We used a mixed-effect logistic regression with uninformative priors (Canessa et al. 2012):

$$
\operatorname{logit}\left(p_{i j}\right)=\underset{\operatorname{pop}(j)}{\propto}+\beta \times \cos \left(\text { date }_{i} \times \frac{2 \pi}{365}-\text { offset }\right)
$$

where $p_{i j}$ is the probability of detecting individual $j$ on date $i$ (calculated as the number of days since 1 January), offset is an estimated parameter used to locate the peak of the cosine function, and $\alpha_{\mathrm{pop}(j)}$ is a populationspecific random intercept that was used to account for between-population variability. We augmented the data with 500 pseudo-individuals randomly allocated between the three populations. Data augmentation consists of adding a number of pseudo-individuals, with allzero capture histories, to the data set, and analyzing a reparameterized version of the same model: this dataexpansion improves model fitting and estimation of the total population size (Royle et al. 2007). We ran the model under the same Markov chain Monte Carlo settings as the prevalence model described above.

We estimated the total size of each population and obtained predictions of the probability of capturing 
each individual across the entire breeding season, from April to September. For all estimated parameters, we evaluated the entire posterior distribution and summarized $95 \%$ credible intervals. We used the estimated distribution of individual probability of capture for each date to predict the number of animals that were predicted to be caught from each population. Using Eq. 4, we also calculated the predicted cumulative proportion of the population that would be caught during the three consecutive surveys that were carried out in 2012.

\section{Predictions for future surveys and validation}

We then used the estimated values for capture probabilities and population sizes, combining the models for prevalence and capture (Eq. 1-3; Supplement), to predict the estimate of prevalence that would be obtained for every day in the 2012 season, in the event that all individuals swabbed returned negative results. We ran the model with three different priors for prevalence: (1) the previously used uninformative prior (uniform 0,1 ), (2) an informative prior with $\alpha=3.5$ and $\beta=31.5$, corresponding to a mean expected prevalence of $0.1 \pm 0.05$ (mean $\pm \mathrm{SD}$ ), on the basis of expert judgment and existing information on the prevalence of chytrid in B. variegata across Europe (Spitzen-van der Sluijs et al. 2010, Sztatecsny and Glaser 2011, Tünde et al. 2012, Canestrelli et al. 2013, Vörös et al. 2013), and (3) an informative prior based on the parameter distribution for prevalence obtained from analyzing the 2011 survey results. To account for stochasticity in the determination of node $n$, we ran each model 1000 times and calculated average results.

Finally, we assessed the predictions of the model by comparing them with the observed results of the 2012 screening surveys. We calculated the proportion of the estimated population caught and compared it with the predictions of the Jolly-Seber model for those dates. We then fitted the post hoc binomial model (Eq. 1-2) to the results of the chytrid swabs to estimate prevalence within each population under the different priors, and compared the results with the predictions previously produced. We assessed the discrepancies between direct estimates and model predictions to evaluate the accuracy of our model in informing the planning of future surveillance programs.

\section{Results}

Between 2011 and 2012, we caught a total of 183 individuals with 403 recaptures (population 1, 70 individuals with 201 recaptures; population 2, 67 individuals with 163 recaptures; population 3, 49 individuals with 59 recaptures). The Jolly-Seber model fitted to the 2011 data showed adequate convergence. The average size of the populations was estimated at 68 (95\% credible intervals; 68-70), 67 (95\% CI; 67-69), and 47 (95\% CI; 47-49) individuals for populations $1-3$, respectively. Daily survival was estimated at 0.95 (95\%
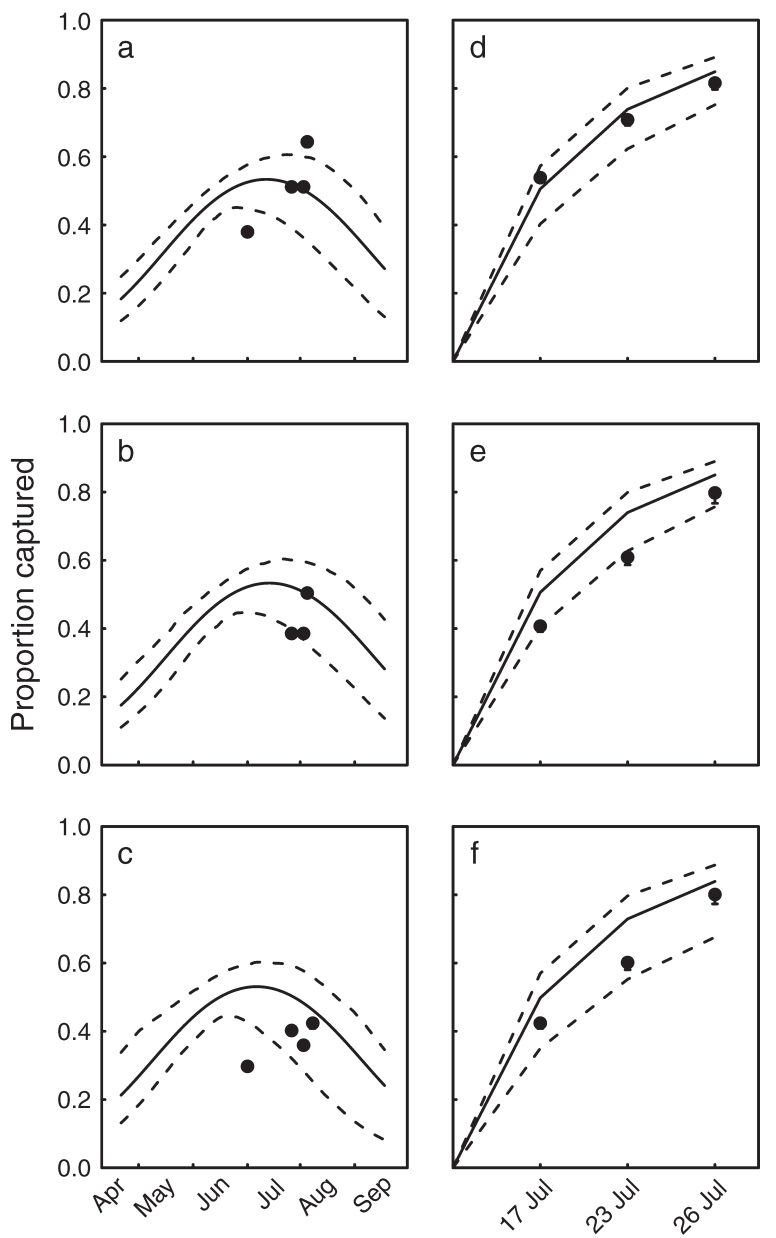

Survey date

FIG. 1. Seasonal variation in recapture rates for Bombina variegata pachypus in the three populations tested (population 1 at top, population 2 in the middle, population 3 at bottom). Panels $(\mathrm{a}-\mathrm{c})$ show probability of individual recapture during individual surveys throughout the season (April-September). Panels (d-f) show cumulative probability of recapture for three consecutive sampling dates in July 2012. Solid lines correspond to model predictions of probability, assumed to equal the proportion of the estimated population size captured on a given date. Points correspond to the actual proportion caught. Dashed lines and error bars correspond to $95 \%$ credible intervals.

CI; 0.94-0.97). The individual probability of capture varied across the season, peaking in the first week of July with a mean maximum value of 0.54 , and was lowest on 11 April, corresponding to the earliest date of the study period, with mean values between 0.18 and 0.22 (Fig. 1). The credible intervals suggested greater variation in the second part of the season (Fig. 1).

When we compared the predictions with the proportion of the estimated population captured in 2012, the first session for populations 1 and 2 (22 June) was overestimated, with the observed value falling outside $95 \%$ credible intervals for populations 1 and 3 (Fig. 1). 

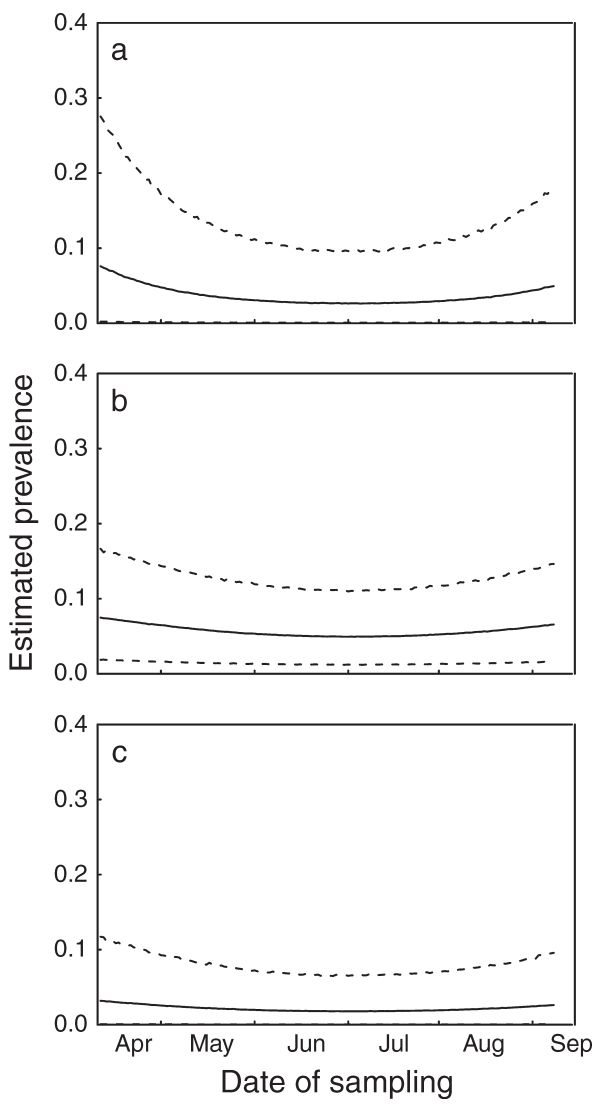

FIG. 2. Predictions of the estimated pathogen prevalence for population 1, assuming no positive individuals were found during surveys on different dates. Plots correspond to predictions made using different priors: (a) uninformative (beta parameters $\alpha=1, \beta=1$ ); (b) informative prior based on existing knowledge $(\alpha=3.5, \beta=31)$; (c) updated prior obtained from analysis of 2011 data $(\alpha=1, \beta=18)$. Dashed lines correspond to $95 \%$ credible intervals.

Surveys in late July were closer to the mean predicted value, with the exception only of the last survey for population 1, which was closer to the upper $95 \%$ credible interval (Fig. 1). The cumulative proportion caught in the three successive surveys in late July was predicted to approach averages of 0.75 and 0.85 for the second and third survey, respectively. We found very close correspondence between the predicted and observed values for population 1 , whereas there was a slight overestimation for populations 2 and 3, particularly for the first two surveys: when comparing credible intervals, predicted and observed values generally showed satisfactory overlap (Fig. 1).

In 2011, we tested 17, 21, and 13 individuals within populations $1-3$. Using the post hoc model with an uninformative prior for the 2011 surveys yielded estimates of $p=0.053$ (95\% CI; 0.001-0.184), $p=$ 0.044 (95\% CI; $0.001-0.157$ ), and $p=0.066$ (95\% CI; $0.002-0.230$ ) for populations $1-3$, respectively. Using the probabilities of capture estimated in the Jolly-Seber model, if no positive individuals were caught during the
2012 season, we predicted the mean estimates of prevalence would be most accurate in correspondence with high capture rates. Overall, the most accurate estimates of prevalence were obtained by maximizing the sample size (surveying when detection was highest), and using prior information regarding prevalence (Fig. 2c). For population 1, when using an uninformative prior, the upper $95 \%$ credible interval decreased from 0.3 in early April to 0.11 in early July (Fig. 2a). This seasonal variation in the uncertainty of estimates decreased when using an informative prior based on existing knowledge, with the upper $95 \% \mathrm{CI}$ decreasing from 0.17 to 0.12 between April and July (Fig. 2b). When the updated prior was used, upper credible intervals remained under 0.1 throughout the season, with estimates always smaller than 0.05 (Fig. 2c). Results were largely similar for other populations.

In 2012, we tested 35, 26, and 17 individuals in the first survey, and 44, 34, and 20 in the second survey, for a cumulative total of 48,40 , and 30 unique individuals. The prevalence estimated from these data was generally close to the predicted values across all combinations of priors and populations (Fig. 3). The realized accuracy of estimates was always at least as narrow as predicted, and using two consecutive surveys almost always provided the expected increase in accuracy, with the only exception of population 2, particularly when using less informative priors: the maximum gain was achieved in population 3, where the upper $95 \%$ confidence interval decreased from $p=0.19$ to $p=0.12$ when adding a second survey and using an uninformative prior.

\section{DisCusSION}

Adequate timing of screening surveys allowed a significant reduction in the uncertainty surrounding estimates of pathogen prevalence. As expected, using a predictive model incorporating population sizes and probabilities of capture helped in identifying the seasonal window for sampling that would maximize the sampling size, resulting in more accurate estimates. Model predictions closely matched the observed results for all populations, suggesting that future screening programs would benefit from accounting for imperfect probability of capture. The incorporation of additional information regarding the expected prevalence of the pathogen (as informative priors) also reduced uncertainty. This partly compensated for the influence of sample size, and therefore improved the accuracy of estimates throughout the season. These results highlight the need for managers to consider the influence of survey efficiency when planning screening programs, and the advantages of using all available and relevant information.

Recent studies have recognized the need to consider uncertainty in wildlife disease monitoring. In particular, McClintock et al. (2010) highlighted the many different sources of uncertainty that can affect inference about wildlife disease, and advocated for a greater incorpora- 
Prior estimate of prevalence

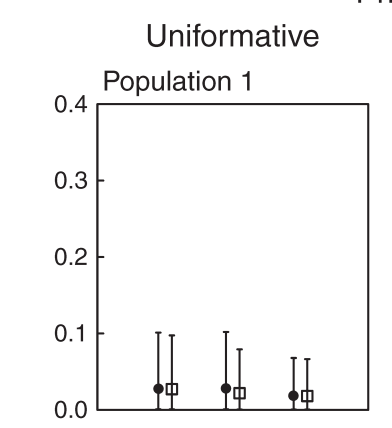

Literature

Updated 2011
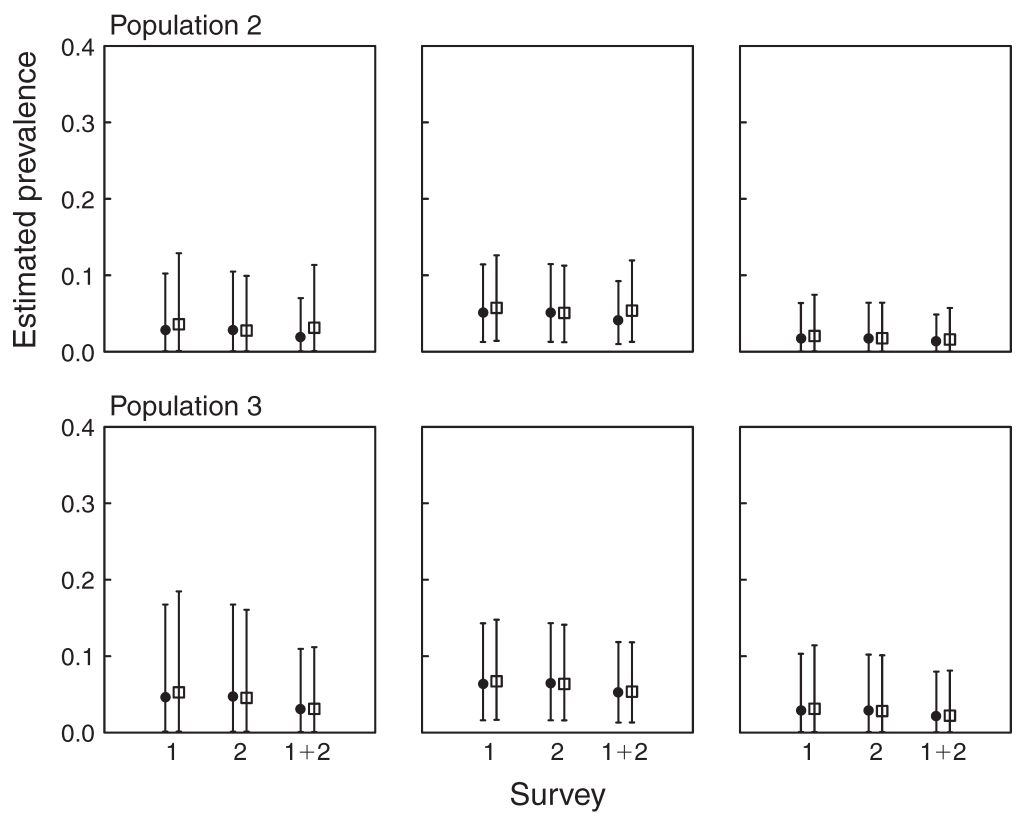

FIG. 3. Estimates of prevalence for each population, as predicted using recapture rates and population sizes (circles) and as observed in the 2012 field surveys (squares). Columns from left to right correspond to different priors used (uninformative, based on existing literature, and updated from the 2011 results). Rows refer to different populations (1, 2, and 3). Within each plot, pairs of points refer to different surveys (22 July [1], 24 July [2], and cumulative results of the two). Error bars indicate $95 \%$ credible intervals.

tion of these concepts in future studies. Our study deals specifically with what McClintock et al. (2010) call sampling uncertainty, in particular, the temporal component of field monitoring, which determines the number of samples collected, and therefore the ability to make precise estimates of prevalence. Alternative model formulations can be used to incorporate examples where there is sampling bias between infected and uninfected individuals, and for false positive error rates (McClintock et al. 2010). Although in this study we assumed perfect sensitivity and specificity of the test procedures, this assumption might be violated, particularly depending on infection intensity of individuals: this different instance of imperfect detection can also be accounted for in estimation techniques (Miller et al. 2012).

Our modeling framework assumes presence of the pathogen (e.g., non-zero prevalence). Therefore, it is not possible to formally infer absence, unless the whole population has been tested. Alternatively, managers might choose to declare pathogen- or disease-free populations when the estimated prevalence falls, with a given confidence, under a selected threshold (Dufour et al. 2001). Research in veterinary science has dealt extensively with the calculation of optimal sample sizes to estimate such freedom from disease (DiGiacomo and Koepsell 1986, Martin et al. 1992, Cameron and Baldock 1998), and the adoption of Bayesian methods has allowed incorporation of prior beliefs about prevalence (Johnson et al. 2004). However, although the chances of $B$. dendrobatidis-induced population declines and losses can realistically be considered smaller when prevalence is low in a population, recent studies have shown that relatively small environmental changes can result in a rapid increase of both prevalence and infection load above the threshold for disease induction, 
including in species commonly considered resistant (Daskin and Alford 2012, Alford 2013, Doddington et al. 2013). On the other hand, the practical difference between absence and low prevalence can be reduced when one considers the likelihood of introduction of the pathogen into open systems. The role of other amphibian species, including introduced ones, as vectors in inducing pathogen and disease spread in vulnerable species has been widely demonstrated in Europe and Italy (Simoncelli et al. 2005, Garner et al. 2006, Spitzenvan der Sluijs et al. 2011).

In this sense, continuous surveillance programs are the most desirable management option, and may prove particularly useful for small, isolated populations where a disease breakout might determine local or broader extinctions (De Castro and Bolker 2005). If programs are managed locally and carried out with continuity, for example, annually, management objectives may include both maximizing their efficiency (increasing sample sizes, and therefore the probability of detecting the pathogen and the accuracy of prevalence estimates), and reducing the cost and effort required to achieve the desired results. In our study, selecting the most suitable time of the year (early July) for screening of $B . v$. pachypus was predicted to reduce the uncertainty surrounding the estimated prevalence, in case no positive results were found, by almost $50 \%$.

It should be noted that, in our model, we assumed that the proportion of the total population that was sampled equaled the individual probability of capture. For our study species, this assumption is justified by the relative tolerance to the pathogen observed in $B$. variegata, where populations are known to have persisted in the presence of chytrid for several years (Canestrelli et al. 2013), suggesting that infected and uninfected individuals will not exhibit important differences in behavior (hence our reference to pathogen prevalence, rather than disease prevalence). However, this assumption may be violated for other species, and lead to different recapture rates: for example, altered behavior may render infected individuals more likely to be captured. When such dynamics are expected, it is possible to account for this bias when estimating either prevalence or recapture and survival rates, for example by using a multiple-state-space formulation (Murray et al. 2009).

The screening sessions themselves can be used to collect data for estimating population size at relatively low cost, particularly where individual recognition can be obtained. Therefore, as screening programs are carried out, priors (both for capture and prevalence) can be updated, if considered necessary. For this study, we underwent considerable sampling effort to collect mark-recapture data for B. v. pachypus to estimate population size and probability of capture. However, these data also provided useful guidance for future screening of the species. The need to carry out such pilot studies, then, should be assessed by managers, trading off the need to collect further information and the quality of future estimates. Local managers of protected areas might already have estimates of population sizes, while capture rates might be estimated more cheaply by repeated counts (Royle 2004), or by expert knowledge.

In addition to selecting the most suitable time or conditions for surveys, the cumulative probability of capture can be increased by carrying out repeated screening sessions. For B. v. pachypus, carrying out two visits in short succession narrowed uncertainty around estimates of prevalence (in the form of credible intervals) by a maximum of $36 \%$ in population 3 when using uninformative priors. The ability to mark and recognize individuals would ensure no unnecessary double samples were carried out, and help to contain costs. However, programs relying on repeat visits will need to consider pathogen dynamics at the screened sites: for example, chytrid prevalence is known to vary seasonally (Kriger and Hero 2007, Berger et al. 2008). The infection status of individuals can also vary between occasions (Berger et al. 2008). Reducing the period between surveys, as in this study, can limit the confounding effect of prevalence fluctuations.

The seasonal variation in prevalence can also complicate the design of sampling protocols: these can be especially obvious when pathogen prevalence and/or load vary in contrast to the probability of capture. For example, for B. v. pachypus in Liguria, we found that individual recapture rates were highest during warm months (end of June-July). For our example populations, located in mountain streams at medium elevation (600 $\mathrm{m}$ above sea level), these periods are also likely to be the most favorable for chytrid, with water temperatures approaching ideal conditions $\left(20-25^{\circ} \mathrm{C}\right.$; Johnson et al. 2003). However, for low-elevation sites in the region, water temperatures in July can exceed $30^{\circ} \mathrm{C}$ and prove unsuitable for the pathogen: in this case, the optimal periods for probability of capture and pathogen prevalence would not coincide, determining a trade-off in surveillance design. Such dynamics could be accounted for in our framework by including date-specific priors for prevalence; optimization might be used to determine the dates most likely to provide maximum accuracy in prevalence estimates.

The adoption of a Bayesian approach in our study facilitated the propagation of parameter uncertainty and allowed the incorporation of prior belief about parameters, particularly regarding pathogen prevalence. Prior information can regard survey-specific and pathogenspecific dynamics (such as seasonal variation in prevalence). For programs with recurring screening, beliefs can be updated or remain constant (for example, where there is a belief in the possibility of invasion). For example, in our study updating our belief in pathogen prevalence, using the narrower posterior distribution from the 2011 screening results decreased the estimated prevalence for the 2012 results, increased precision, and 
made the quality of inference almost independent of variable probabilities of capture.

Prompt detection of new infections is likely to be the most difficult component of chytrid monitoring, given the uncertainty in detection of both individuals and the pathogen. We have shown how simple models of survey efficiency and estimation of prevalence can be integrated to design programs that maximize the information that can be obtained from field surveys. Adequate planning of surveillance programs on the basis of quantitative information can help allocate resources and ensure that adequate conservation actions are taken rapidly.

\section{AcKNowledgments}

We thank F. Belfiore, G. Canessa, M. Guerrina, A. Morán Ordóñez, L. Noja, and P. Piana for assistance with field surveys, and G. Guillera-Arroita, J. J. Lahoz-Monfort, M. McCarthy, and W. Morris for help with statistical analyses. Comments by G. Heard, S. Salvidio, J. Weiss, and two anonymous referees greatly improved the original manuscript. Capture and handling permits were granted by the Ministero dell'Ambiente e della Tutela del Territorio (DPN-2010-370, 12-01-2010). Fieldwork was partly funded by the 2011 Grant in Herpetology by the Societas Europaea Herpetologica. Manuscript preparation was supported by funding from the University of Melbourne and the ARC Centre of Excellence for Environmental Decisions.

\section{Literature Cited}

Alford, R. A. 2013. Environmental epidemiology: fluctuating temperature effects. Nature Climate Change 3:101-103.

Andreone, F., C. Corti, R. Sindaco, A. Romano, F. Giachi, S. Vanni, and G. Delfino. 2009. Bombina pachypus. In IUCN 2011. IUCN Red List of Threatened Species. Version 2011.2. www.iucnredlist.org

Barandun, J., and H. U. Reyer. 1998. Reproductive ecology of Bombina variegata: habitat use. Copeia 1998:497-500.

Barandun, J., H. U. Reyer, and B. Anholt. 1997. Reproductive ecology of Bombina variegata: aspects of life history. Amphibia-Reptilia 18:347-355.

Barbieri, F., F. Bernini, F. M. Guarino, and A. Venchi. 2004. Distribution and conservation status of Bombina variegata in Italy (Amphibia, Bombinatoridae). Bollettino di Zoologia 71:83-90.

Berger, L., et al. 1998. Chytridiomycosis causes amphibian mortality associated with population declines in the rain forests of Australia and Central America. Proceedings of the National Academy of Sciences USA 95:9031-9036.

Berger, L., et al. 2008. Effect of season and temperature on mortality in amphibians due to chytridiomycosis. Australian Veterinary Journal 82:434-439.

Brooks, S. P., and A. Gelman. 1998. General methods for monitoring convergence of iterative simulations. Journal of Computational and Graphical Statistics 7:434-455.

Burgman, M., B. Wintle, C. Thompson, A. Moilanen, M. Runge, and Y. Ben-Haim. 2009. Reconciling uncertain costs and benefits in Bayes nets for invasive species management. Risk Analysis 30:277-284.

Cameron, A. R., and F. C. Baldock. 1998. A new probability formula for surveys to substantiate freedom from disease. Preventive Veterinary Medicine 34:1-17.

Canessa, S., G. W. Heard, K. M. Parris, and M. A. McCarthy. 2012. Integrating variability in detection probabilities when designing wildlife surveys: a case study of amphibians from south-eastern Australia. Biodiversity and Conservation 21:729-744.
Canessa, S., A. Martel, and F. Pasmans. 2013a. No detection of chytrid in first systematic screening of Bombina variegata pachypus (Anura: Bombinatoridae) in Liguria, northern Italy. Acta Herpetologica 8:59-63.

Canessa, S., F. Oneto, D. Ottonello, A. Arillo, and S. Salvidio. 2013b. Land abandonment may reduce disturbance and affect the breeding sites of an endangered amphibian in northern Italy. Oryx 47:280-287.

Canestrelli, D., M. Zampiglia, and G. Nascetti. 2013. Widespread occurrence of Batrachochytrium dendrobatidis in contemporary and historical samples of the endangered Bombina pachypus along the Italian peninsula. PloS ONE 8:e63349.

Daskin, J. H., and R. A. Alford. 2012. Context-dependent symbioses and their potential roles in wildlife diseases. Proceedings of the Royal Society B 279:1457-1465.

De Castro, F., and B. Bolker. 2005. Mechanisms of diseaseinduced extinction. Ecology Letters 8:117-126.

DiGiacomo, R. F., and T. D. Koepsell. 1986. Sampling for detection of infection or disease in animal populations. Journal of the American Veterinary Medical Association 189:22-23.

Doddington, B. J., J. Bosch, J. A. Oliver, N. C. Grassly, G. Garcia, B. R. Schmidt, T. W. Garner, and M. C. Fisher. 2013. Context-dependent amphibian host population response to an invading pathogen. Ecology 94:1795-1804.

Dufour, B., R. Pouillot, and B. Toma. 2001. Proposed criteria to determine whether a territory is free of a given animal disease. Veterinary Research 32:545-563.

Fisher, M. C., T. W. J. Garner, and S. F. Walker. 2009. Global emergence of Batrachochytrium dendrobatidis and amphibian chytridiomycosis in space, time, and host. Annual Review of Microbiology 63:291-310.

Gagliardo, R., P. Crump, E. Griffith, J. Mendelson, H. Ross, and K. Zippel. 2008. The principles of rapid response for amphibian conservation, using the programmes in Panama as an example. International Zoo Yearbook 42:125-135.

Garner, T. W. J., M. W. Perkins, P. Govindarajulu, D. Seglie, S. Walker, A. A. Cunningham, and M. C. Fisher. 2006. The emerging amphibian pathogen Batrachochytrium dendrobatidis globally infects introduced populations of the North American bullfrog, Rana catesbeiana. Biology Letters 2:455459.

Garrard, G. E., S. A. Bekessy, M. A. McCarthy, and B. A. Wintle. 2008. When have we looked hard enough? A novel method for setting minimum survey effort protocols for flora surveys. Austral Ecology 33:986-998.

Guarino, F., O. Picariello, and A. Venchi. 2007. Bombina pachypus (Bonaparte, 1838). Pages 277-280 in C. Corti, M. Capula, L. Luiselli, R. Sindaco, and E. Razzetti, editors. Amphibia. Fauna d'Italia. Calderini, Bologna, Italy.

Hartel, T. 2008. Movement activity in a Bombina variegata population from a deciduous forested landscape. NorthWestern Journal of Zoology 4:79-90.

Hartel, T., S. Nemes, and G. Mara. 2007. Breeding phenology and spatio-temporal dynamics of pond use by the yellowbellied toad (Bombina variegata) population: the importance of pond availability and duration. Acta Zoologica Lituanica 17:56-63.

Johnson, M. L., L. Berger, L. Phillips, and R. Speare. 2003. Fungicidal effects of chemical disinfectants, UV light, desiccation and heat on the amphibian chytrid, Batrachochytrium dendrobatidis. Diseases of Aquatic Organisms 57:255260.

Johnson, W. O., C. L. Su, I. A. Gardner, and R. Christensen. 2004. Sample size calculations for surveys to substantiate freedom of populations from infectious agents. Biometrics 60:165-171. 
Kéry, M., and M. Schaub. 2011. Bayesian population analysis using WinBUGS: a hierarchical perspective. Academic Press, Burlington, Massachusetts, USA.

Kriger, K. M., and J. M. Hero. 2007. Large-scale seasonal variation in the prevalence and severity of chytridiomycosis. Journal of Zoology 271:352-359.

Lachish, S., A. M. Gopalaswamy, S. C. L. Knowles, and B. C. Sheldon. 2012. Site-occupancy modelling as a novel framework for assessing test sensitivity and estimating wildlife disease prevalence from imperfect diagnostic tests. Methods in Ecology and Evolution 3:339-348.

Lunn, D. J., A. Thomas, N. Best, and D. Spiegelhalter. 2000. WinBUGS - a Bayesian modelling framework: concepts, structure, and extensibility. Statistics and Computing 10:325-337.

MacKenzie, D. I., and W. L. Kendall. 2002. How should detection probability be incorporated into estimates of relative abundance? Ecology 83:2387-2393.

Martin, S. W., M. Shoukri, and M. A. Thorburn. 1992. Evaluating the health status of herds based on tests applied to individuals. Preventive Veterinary Medicine 14:33-43.

McClintock, B. T., J. D. Nichols, L. L. Bailey, D. I. MacKenzie, W. Kendall, and A. B. Franklin. 2010. Seeking a second opinion: uncertainty in disease ecology. Ecology Letters 13:659-674.

Miller, D. A. W., B. L. Talley, K. R. Lips, and E. H. Campbell Grant. 2012. Estimating patterns and drivers of infection prevalence and intensity when detection is imperfect and sampling error occurs. Methods in Ecology and Evolution 3:850-859.

Murray, K. A., L. F. Skerratt, R. Speare, and H. McCallum. 2009. Impact and dynamics of disease in species threatened by the amphibian chytrid fungus, Batrachochytrium dendrobatidis. Conservation Biology 23:1242-1252.

Plummer, M. 2003. JAGS: a program for analysis of Bayesian graphical models using Gibbs sampling. In K. Hornik, F. Leisch, and A. Zeileis, editors. Proceedings of the 3rd International Workshop on Distributed Statistical Computing (DSC 2003), Vienna, Austria, March 20-22, 2003. R Foundation for Statistical Computing, Vienna, Austria.
Royle, J. A. 2004. $N$-mixture models for estimating population size from spatially replicated counts. Biometrics 60:108-115.

Royle, J. A., R. M. Dorazio, and W. A. Link. 2007. Analysis of multinomial models with unknown index using data augmentation. Journal of Computational and Graphical Statistics 16:67-85.

Schmidt, B. 2004. Declining amphibian populations: the pitfalls of count data in the study of diversity, distribution, dynamics and demography. Herpetological Journal 14:167-174.

Simoncelli, F., A. Fagotti, R. Dall'Olio, D. Vagnetti, R. Pascolini, and I. Di Rosa. 2005. Evidence of Batrachochytrium dendrobatidis infection in water frogs of the Rana esculenta complex in central Italy. EcoHealth 2:307-312.

Spitzen-van der Sluijs, A., A. Martel, E. Wombwell, P. Van Rooij, R. Zollinger, T. Woeltjes, M. Rendle, F. Haesebrouck, and F. Pasmans. 2011. Clinically healthy amphibians in captive collections and at pet fairs: a reservoir of Batrachochytrium dendrobatidis. Amphibia-Reptilia 32:419-423.

Spitzen-van der Sluijs, A., R. Zollinger, W. Bosman, F. Pasmans, A. Martel, P. Van Rooij, and F. Clare. 2010. Short report: Batrachochytrium dendrobatidis in amphibians in the Netherlands and Flanders (Belgium). Ravon, Nijmegen, Netherlands.

Stagni, G., R. Dall'Olio, U. Fusini, S. Mazzotti, C. Scoccianti, and A. Serra. 2004. Declining populations of apennine yellow-bellied toad Bombina pachypus in the northern Apennines (Italy): is Batrachochytrium dendrobatidis the main cause? Italian Journal of Zoology 71:151-154.

Sztatecsny, M., and F. Glaser. 2011. From the eastern lowlands to the western mountains: first records of the chytrid fungus Batrachochytrium dendrobatidis in wild amphibian populations from Austria. Herpetological Journal 21:87-90.

Tünde, G. J., K. Szabó, and J. Vörös. 2012. Survey on Batrachochytrium dendrobatidis in an amphibian community in Bakony Mountains, Hungary. Állattani Közlemények 97:47-59.

Vörös, J., J. Bosch, Á. Dán, and T. Hartel. 2013. First record of Batrachochytrium dendrobatidis on amphibians in Romania. North-Western Journal of Zoology 9:446-449.

\section{Supplemental Material}

\section{Supplement}

BUGS/JAGS code and instructions for estimation of pathogen prevalence and for Jolly-Seber model to estimate population size and recapture rates (Ecological Archives A024-070-S1). 